\title{
Lipoprotein X in Cholestasis and Questionable Atherogenic Risk
}

\author{
Ana Paula Marte Chacra ${ }^{1}$, Anita LR Saldanha ${ }^{2}$, Raul D Santos Filho ${ }^{1}$ and Tania Leme da Rocha Martínez ${ }^{1,2 *}$ \\ ${ }^{1}$ Department of Medicine, University of São Paulo, Brazil \\ ${ }^{2}$ Department of Nephrology, BP - The Portuguese Beneficence of São Paulo, Brazil
}

*Corresponding author: Tania Leme da Rocha Martinez, Department of Nephrology, BP - The Portuguese Beneficence of São Paulo, Brazil.
Received Date: May 21, 2020

Published Date: June 08, 2020

\begin{abstract}
Lipoprotein $\mathrm{X}$ is still an enigma for its lack of evidence as a risk association with atherosclerosis. Primary biliary cirrhosis is a chronic, autoimmune cholestatic liver disease characterized by the presence of T cells that destroy the biliary epithelium. In patients with primary biliary cirrhosis, total cholesterol levels may reach values between 500 and $1000 \mathrm{mg} / \mathrm{dL}$, with cutaneous xanthomas similar to those observed in genetic hypercholesterolemias. The rise in cholesterol is largely due to increased serum levels of lipoprotein-X. It has a small amount of albumin, apoprotein $\mathrm{AI}$, apoprotein Cs and apoprotein $\mathrm{E}$ and has no apoprotein B. The formation of lipoprotein-X results from regurgitation of bile lipids in plasma, as well as the accumulation of plasma phospholipids and free cholesterol, resulting from the lower activity of lecithin: acyltransferase cholesterol. Multicenter randomized trials are needed to assess the impact of lipid-lowering patients on lipid reduction and cardiovascular risk, as well as the effect on hepatic cholestasis. Treatment with statins is not indicated for lowering cholesterol in patients with primary biliary cirrhosis and dyslipidemia, as there is no evidence that these patients have high cardiovascular risk.
\end{abstract}

Keywords: Atherosclerosis; Cholesterol; Lecithin cholesterol acyl transferase; Lipoprotein X; Primary Biliary cirrhosis

Abbreviations: LCAT: Lecithin Cholesterol Acyl Transferase; LDL: Low Density Lipoprotein; LDL-c: Low Density Lipoprotein Cholesterol; LP-X: Lipoprotein X; PBC: Primary Biliary Cirrhosis

\section{Introduction}

Cholestatic Liver Diseases among the cholestatic diseases of the liver, Primary Biliary Cirrhosis (PBC), although rare, is very representative of a secondary cause of dyslipidemia. $\mathrm{PBC}$ is a chronic, autoimmune cholestatic liver disease characterized by the presence of T cells that destroy the biliary epithelium [1]. The average annual incidence ranges from 1.6 to 14.6 cases per 100,000 people, respectively [2]. PBC affects more women than men, in the ratio of 8:1 [3]. The mean age of diagnosis is around 66 years [4]. The disease can be divided into four phases[4]: an asymptomatic phase lasting up to 20 years; symptomatic from 5 to 10 years, in which the patient is anicteric or only slightly jaundiced; short preterminal characterized by severe jaundice; and finally terminal liver failure [4]. Hypercholesterolemia in PBC: in patients with PBC, total cholesterol levels can reach values between 500 and $1000 \mathrm{mg} / \mathrm{dL}$, with cutaneous xanthomas similar to those observed in genetic hypercholesterolemias. In these patients, the increase in serum cholesterol levels is largely due to the increased level of lipoprotein-X (LP-X), which is a lipoprotein with a lower density than LDL, has no apoprotein B in its structure and is enriched with free cholesterol and phospholipids [5]. The formation of LP-X results from regurgitation of bile lipids in plasma, as well as the accumulation of plasma phospholipids and free cholesterol, resulting from lower lecithin activity: acyltransferase cholesterol (LCAT) [5,6]. LP-X has been shown to have antiatherogenic properties, such as inhibition of oxidation of LDL-c particles [7]. In 
this context, patients with PBC who have high cholesterol due to the increase in LP-X may have attenuated cardiovascular risk.

Few studies have evaluated cardiovascular events in patients with dyslipidemia secondary to $\mathrm{PBC}$, since the disease has a rare prevalence. Only one observational study demonstrated cardiovascular mortality of $12 \%$ in this population [8]. These results pointed to a possible role of statins in reducing cardiovascular risk in these patients. Case reports suggest that statins, in addition to reducing LDL-c and triglyceride levels, reduce intrahepatic cholestasis markers, with improved liver function [9-11]. Multicenter randomized trials are needed to assess the impact of lipid-lowering patients on lipid reduction and cardiovascular risk, as well as the effect on hepatic cholestasis. Treatment with statins is not indicated for lowering cholesterol in patients with PBC and dyslipidemia, as there is no evidence that these patients have high cardiovascular risk.

\section{Acknowledgement}

None.

\section{Conflict of Interest}

No conflict of interest.

\section{References}

1. Kaplan MM, Gershwin ME (2005) Primary biliary cirrhosis. N Engl J Med 353(12): 1261-1273

2. Metcalf JV, Bhopal RS, Gray J, Howel D, James OF (1997) Incidence and prevalence of primary biliary cirrhosis in the city of Newcastle upon Tyne, England. Int J Epidemiol 26(4): 830-836.
3. Boberg KM, Aadland E, Jahnsen J, Raknerud N, Stiris M, et al. (1998) Incidence and prevalence of primary biliary cirrhosis, primary sclerosing cholangitis, and autoimmune hepatitis in a Norwegian population. Scand J Gastroenterol 33(1): 99-103.

4. Parés A, Rodés J (2003) Natural history of primary biliary cirrhosis. Clin Liver Dis 7(4): 779-794.

5. Manzato E, Fellin R, Baggio G, Walch S, Neubeck W, et al. (1976) Formation of lipoprotein-X. Its relationship to bile compounds. J Clin Invest 57(5): 1248-1260.

6. Jahn CE, Schaefer EJ, Taam LA, Hoofnagle JH, Lindgren FT, et al (1985) Lipoprotein abnormalities in primary biliary cirrhosis. Association with hepatic lipase inhibition as well as altered cholesterol esterification. Gastroenterology 89(6): 1266-1278.

7. Chang PY, Lu SC, Su TC, Chou SF, Huang WH, et al. (2004) Lipoprotein-X reduces LDL atherogenicity in primary biliary cirrhosis by preventing LDL oxidation. J Lipid Res 45(11): 2116-2122.

8. Van Dam GM, Gips CH (1997) Primary biliary cirrhosis in The Netherlands. An analysis of associated diseases, cardiovascular risk, and malignancies on the basis of mortality figures. Scand J Gastroenterol 32(1): 77-83.

9. Ritzel U, Leonhardt U, Näther M, Schäfer G, Armstrong VW, et al. (2002) Simvastatin in primary biliary cirrhosis: effects on serum lipids and distinct disease markers. J Hepatol 36(4): 454-458.

10. Kamisako T, Adachi Y (1995) Marked improvement in cholestasis and hypercholesterolemia with simvastatin in a patient with primary biliary cirrhosis. Am J Gastroenterol 90(7): 1187-1188.

11. Kurihara T, Akimoto M, Abe K, Ishiguro H, Niimi A, et al. (1993) Experimental use of pravastatin in patients with primary biliary cirrhosis associated with hypercholesterolemia. Clin Ther 15(5): 890898. 